\title{
Cuidados de enfermagem relacionados à prevenção de prolapso em ostomias intestinais: uma revisão integrativa da literatura
}

\author{
Nursing care related to the prevention of prolapse in intestinal ostomies: an integrative literature \\ review
}

Atención de enfermería relacionada con la prevención del prolapso en las ostomías intestinales: revisión integradora de la literatura

\section{Resumo}

Objetivo: Identificar os principais cuidados de enfermagem relacionados à prevenção de prolapso em ostomias intestinais. Metodologia: Trata-se de uma revisão integrativa, utilizando as bases de dados Literatura Latino-americana e do Caribe em Ciências da Saúde (LILACS), Scientific Electronic Library Online (SciELO), Base de Dados de Enfermagem (BDENF), Medical Literature Analysis and Retrieval System On-line (PubMed/MedLine) e Cumulative Index to Nursing and Allied Health Literature (CINAHL), nos idiomas português, inglês e espanhol, publicados entre 2011 e 2020. Após a busca na literatura científica, foram selecionados vinte e dois estudos para análise sobre os cuidados relacionados à prevenção do prolapso. Resultados: A demarcação do estoma, a educação pré-operatória, o acompanhamento telefônico educacional de suporte pós-alta realizado pela estomaterapeuta foram apontados como cuidados e/ou estratégias para prevenção do prolapso. Conclusão: A demarcação cirúrgica prévia do estoma associada à educação pré-operatória e aos programas de educação e/ou intervenção voltados para o gerenciamento da ostomia no pós-operatório podem ser consideradas importantes tecnologias de cuidado capazes de prevenir o prolapso em ostomias intestinais. Todavia, a realização da demarcação ainda carece de evidências científicas mais robustas, tendo em vista que sua prática ainda não é efetivada, em larga escala, no âmbito internacional.

Palavras-chave: Ostomia; Complicações pós-operatórias; Prolapso; Cuidados de enfermagem; Estomaterapia.

\section{Abstract}

Objective: To identify the main nursing care related to the prevention of prolapse in intestinal ostomies. Methodology: This is an integrative review, using the databases of Literatura Latino-americana e do Caribe em Ciências da Saúde (LILACS), Scientific Electronic Library Online (SciELO), Base de Dados de Enfermagem (BDENF), Medical 
Literature Analysis and Retrieval System On-line (PubMed/MedLine) and Cumulative Index to Nursing and Allied Health Literature (CINAHL), in Portuguese, English and Spanish, published between 2011 and 2020. After searching the scientific literature, twenty-two studies were selected for analysis on the care related to the prevention of prolapse. Results: The demarcation of the stoma, preoperative education, educational telephone follow-up of post-discharge support carried out by the stomatherapist were appointed as care and/or strategies for preventing prolapse. Conclusion: Previous surgical demarcation of the stoma associated with preoperative education and education and/or intervention programs aimed at postoperative ostomy management can be considered important care technologies capable of preventing prolapse in intestinal ostomies. However, the realization of demarcation still lacks more robust scientific evidence, considering that its practice is still not carried out, on a large scale, in the international scope.

Keywords: Ostomy; Postoperative complications; Prolapse; Nursing care; Enterostomal therapy.

\section{Resumen}

Objetivo: Identificar los principales cuidados de enfermería relacionados con la prevención del prolapso en las ostomías intestinales. Metodología: Se trata de una revisión integradora, utilizando las bases de datos de Literatura Latinoamericana e do Caribe em Ciências da Saúde (LILACS), Scientific Electronic Library Online (SciELO), Base de Dados de Enfermagem (BDENF), Medical Literature Analysis and Retrieval System On-line (PubMed/MedLine) y Cumulative Index to Nursing and Allied Health Literature (CINAHL), en portugués, inglés y español, publicado entre 2011 y 2020. Luego de buscar en la literatura científica, se seleccionaron veintidós estudios para analizar los cuidados relacionados con la prevención del prolapso. Resultados: La demarcación del estoma, la educación preoperatoria, el seguimiento telefónico educativo para el apoyo post-alta realizado por el terapeuta de ostomía fueron designados como cuidados y / o estrategias para prevenir el prolapso. Conclusión: La demarcación quirúrgica previa del estoma asociada a la educación preoperatoria y los programas de educación y / o intervención dirigidos al manejo de la ostomía posoperatoria pueden considerarse tecnologías de atención importantes capaces de prevenir el prolapso en las ostomías intestinales. Sin embargo, la realización de la demarcación aún carece de evidencia científica más robusta, considerando que su práctica aún no se lleva a cabo, a gran escala, en el ámbito internacional.

Palabras clave: Estomía; Complicaciones posoperatorias; Prolapso; Atención de enfermería; Estomaterapia.

\section{Introdução}

Ostomia ou estomia é, por definição, a exteriorização de órgãos ou vísceras para o meio externo através da parede abdominal ou a partir de outros órgãos, para fins de drenagem, eliminação ou nutrição. Em se tratando das ostomias intestinais, sua finalidade é a eliminação de efluentes fecais, podendo ser classificadas em ileostomias, quando confeccionadas no íleo, e colostomias, quando inseridas no intestino grosso. Quanto ao tempo de permanência classificam-se em temporária ou definitiva e sua reversão depende da patologia que motivou sua confecção, bem como, do prognóstico da doença e de outros critérios clínicos (Nascimento et al., 2018).

Uma ostomia muda significativamente a vida de uma pessoa, haja vista as mudanças nas dimensões física, psicológica e social que impactam diretamente a qualidade de vida (Byfield, 2020). Nesse contexto, as evidências científicas sugerem haver uma estreita relação entre as complicações decorrentes do estoma e a deterioração da autoimagem, uma vez que o indivíduo necessitará desenvolver habilidades para conviver com o corpo alterado, a exemplo do uso e troca da bolsa coletora (Giordano et al., 2020). Além disso, a pessoa ostomizada poderá se sentir mutilada, com baixa autoestima, e manifestar sentimentos de inutilidade e autoeficácia reduzida (De Melo et al., 2021).

Convém ressaltar que após a confecção do estoma é indispensável o treinamento dos pacientes quanto ao uso dos dispositivos necessários para cada tipo de estoma. Esses equipamentos são as bolsas coletoras de sistema único ou composto, que são descartáveis, aderem à pele e ao redor do estoma (Duluklu \& Çelik, 2020). Há também os adjuvantes que são utilizados para proteger a pele, por exemplo as barreiras protetoras em forma de pó, pasta ou placa, visam prevenir a oxidação da pele em virtude do contato direto com os efluentes liberados pelo estoma (Silva et al., 2020).

Como qualquer procedimento cirúrgico, a ostomia está associada à ocorrência de complicações precoces e tardias. Dentre elas o prolapso se destaca como uma complicação tardia que pode estar associada à localização inadequada do estoma, à ausência da demarcação do estoma no pré-operatório, à técnica cirúrgica empregada, à mobilização excessiva da alça intestinal e a fatores de risco como obesidade, pressão intra-abdominal elevada, entre outros (Sobrado Júnior et al., 2020). Essa 
complicação consiste na exteriorização do segmento de alça intestinal através do estoma e, geralmente, está associada à hérnia paraestomal. Estima-se que o prolapso ocorre em 8 a $75 \%$ das ileostomias, sendo menos frequente nas colostomias ( 2 a $18 \%$ ) (Ambe et al., 2018).

Na perspectiva da reabilitação do paciente que convive com uma ostomia, o enfermeiro possui um papel primordial no processo de adaptação do indivíduo ao estoma, bem como, no manejo e na prevenção de complicações pós-operatórias. Destarte, os cuidados com a ostomia devem ser planejados e implementados desde o pré até o pós-operatório e devem contemplar além do cuidado direto com o estoma, os aspectos nutricionais, comportamentais e sociais (O’Flynn, 2018).

No pré-operatório, o estomaterapeuta possui competência técnica para realizar a demarcação do estoma, além de esclarecer ao paciente quanto ao seu processo saúde-doença, vida social e trabalhista, hábito alimentar, diagnóstico, a cirurgia definida pelo cirurgião, sua finalidade e qual o significado de viver com uma ostomia (Kim et al., 2021). No pós-operatório, esse especialista avalia a confecção, localização e condição do estoma, sua funcionalidade, risco de complicações precoces e tardias, orienta o cuidado com o estoma e manejo com os dispositivos e adjuvantes, além de preparar o indivíduo para alta hospitalar (Almeida et al., 2021).

Entretanto, embora a literatura realce a demarcação do estoma como um cuidado importante na prevenção do prolapso, sabe-se que a ocorrência dessa complicação está associada a fatores diversos que escapam à capacidade de gerenciamento da enfermagem e do cirurgião do aparelho digestivo, haja vista que o prolapso pode ser decorrente de uma iatrogenia, de uma avaliação perioperatória ineficaz, como também, pode ser resultado de uma confluência de fatores intrínsecos e extrínsecos do paciente associados ao procedimento cirúrgico (Zelga et al., 2021).

Nesse contexto, vale ressaltar a lacuna na produção de conhecimento científico acerca dos cuidados de enfermagem no período pré, trans e pós-operatório voltados à prevenção de prolapso em ostomias intestinais, visto que a maioria dos estudos enfatiza a prevenção quaternária das complicações e não a prevenção secundária. Atrelado a esse fato, até o desenvolvimento dessa revisão, não foi encontrada na literatura ferramentas do tipo algoritmos, softwares de gestão ou aplicativos voltados, especificamente, à prevenção do prolapso em ostomias intestinais (Heerschap \& Butt, 2021).

Este estudo justifica-se pela necessidade de obtenção de estudos que retratem, categoricamente, os cuidados de enfermagem direcionados à prevenção do prolapso em ostomias intestinais. Sabe-se que a demarcação prévia do estoma possibilita uma melhor recuperação e adaptação ao paciente, reduz o tempo de internação e, consequentemente, otimiza os gastos públicos (Estrada et al., 2021), porém outros cuidados devem ser investigados, a fim de serem incorporados à prática clínica da Estomaterapia. Perante ao exposto, o presente estudo tem como objetivo identificar os principais cuidados de enfermagem relacionados à prevenção de prolapso em ostomias intestinais.

\section{Metodologia}

Trata-se de um estudo metodológico do tipo revisão integrativa, o qual é caracterizado pela capacidade de agrupamento e síntese das evidências científicas relevantes sobre determinado assunto ou questão norteadora. Esse tipo de estudo exige padrões de rigor, clareza, replicação e possui seis etapas para sua concretização (Moura et al., 2015). As etapas estão descritas a seguir.

$1^{\text {a }}$ etapa: resultou na elaboração da seguinte pergunta de pesquisa: Quais são os cuidados de enfermagem relacionados à prevenção de prolapso em ostomias intestinais? Para tanto, utilizou-se a estratégia PICO (acrônimo para patient, intervention, comparison, outcomes). P: (colostomy OR ostomy OR enterostomy OR postoperative complications OR prolapse); I: (nursing care OR stoma demarcation OR devices; C: (Não se aplica); O: (Secondary Prevention OR Adaptation). Destarte, a pergunta de pesquisa: 
$2^{\mathrm{a}}$ etapa: Para a consulta dos estudos foram utilizadas as bases de dados Literatura Latino-americana e do Caribe em Ciências da Saúde (LILACS), Scientific Electronic Library Online (SciELO), Base de Dados de Enfermagem (BDENF), Medical Literature Analysis and Retrieval System On-line (PubMed/MedLine) e Cumulative Index to Nursing and Allied Health Literature (CINAHL). Utilizou-se os termos Medical Subject Headlings (MeSH) e Descritores em Ciências da Saúde (DeCS), adaptados para cada base de dados e combinados por meio do operador booleano OR e AND. O levantamento da produção do conhecimento ocorreu nos meses de fevereiro, março, abril e maio de 2021.

Os critérios de inclusão foram: estudos primários, envolvendo pesquisa com seres humanos, publicados entre 2011 e 2020 que dissertam sobre o tema, tais como: cuidados de enfermagem, cuidados com a colostomia, prevenção de prolapso, prevenção secundária, troca de bolsa de colostomia, estomaterapeuta, adjuvantes para colostomia, demarcação do estoma e assistência pré-operatória de enfermagem, publicados nos idiomas português, inglês e espanhol. Quanto aos critérios de exclusão foram considerados os artigos duplicados, estudos secundários (revisão) e aqueles que não responderam à pergunta de pesquisa.

$3^{\text {a }}$ etapa: A estratégia PICO foi compartilhada em um formulário do Google Forms, que possibilitou a contribuição simultânea dos autores. Com o auxílio do programa Rayyan desenvolvido pelo Qatar Computing Research Institute (QCRI) versão 0.1.0, que permite a inclusão e exclusão dos artigos e sua respectiva blindagem (Ouzanni et al., 2016). Dois pesquisadores, de forma independente, para evitar vieses na seleção dos artigos, realizaram a avaliação inicialmente pelos títulos relevantes, depois pelos resumos e, por fim, pelo texto completo. As discrepâncias encontradas foram analisadas por consenso entre os revisores, com a participação de um terceiro revisor quando necessário. A estatística Kappa de Cohen foi empregada para medir a confiabilidade entre os avaliadores (Warrens, 2021).

$4^{\mathrm{a}}$ etapa: Para a coleta de dados dos artigos utilizou-se o Instrumento de Ursi (Ursi \& Galvão, 2006) adaptado pelos autores, a partir do qual foram extraídas as seguintes informações: autor, ano, país, periódico, objetivo, método, seleção da amostra, nível de evidência, cuidados de enfermagem, resultados.

$5^{\mathrm{a}}$ etapa: procedeu-se à interpretação e síntese dos resultados. Foram comparados os dados evidenciados na análise dos artigos e descritas as lacunas do conhecimento, como também, prioridades para estudos posteriores. As limitações do estudo também foram apresentadas.

$6^{a}$ etapa: a apresentação da revisão foi expressa em figura e quadros, na qual evidenciou-se o autor, ano, país, periódico, objetivo, método, seleção da amostra, nível de evidência, cuidados de enfermagem, resultados, sendo possível a comparação entre todos os estudos selecionados e, consecutivamente, a identificação de padrões, diferenças e a sublocação desses tópicos como parte integrante da discussão geral.

Para avaliar a qualidade metodológica dos estudos, utilizou-se a pirâmide dos sete níveis de evidência (Melnyk et al., 2014), conforme demonstrado, no Quadro 1, a seguir.

Quadro 1 - Correlação entre o nível de evidência e o delineamento metodológico do estudo.

\begin{tabular}{|l|c|}
\hline \multicolumn{1}{|c|}{ Descrição } & Nível de Evidência \\
\hline $\begin{array}{l}\text { Evidências provenientes de revisão sistemática ou metanálise de todos relevantes ensaios } \\
\text { clínicos randomizados controlados ou oriundas de diretrizes clínicas baseadas em revisões } \\
\text { sistemáticas de ensaios clínicos randomizados controlados }\end{array}$ & I \\
\hline $\begin{array}{l}\text { Evidências derivadas de pelo menos um ensaio clínico randomizado controlado bem } \\
\text { delineado }\end{array}$ & II \\
\hline Evidências obtidas de ensaios clínicos bem delineados sem randomização & III \\
\hline Evidências provenientes de estudos de coorte e de caso-controle bem delineados & IV \\
\hline Evidências originárias de revisão sistemática de estudos descritivos e qualitativos & V \\
\hline Evidências derivadas de um único estudo descritivo ou qualitativo & VI \\
\hline Evidências oriundas de opinião de autoridades e/ou relatório de comitês de especialistas & VII \\
\hline
\end{tabular}

Fonte: Melnyk et al. (2014). 


\section{Resultados}

Inicialmente, foram encontradas 6678 publicações indexadas às bases de dados. Após concluídas todas as etapas de seleção, foram incluídos no estudo 22 artigos, conforme detalhado na Figura 1. Os cálculos do índice Kappa verificaram concordância entre os autores na seleção dos estudos incluídos com nível de confiabilidade de 0,91 .

Figura 1 - Fluxograma da seleção dos estudos recuperados nas bases de dados, adaptado do Preferred Reporting Items for Systematic Review and Meta-Analyses (PRISMA) (Page et al., 2021). Aracaju, SE, Brasil, 2021.

\begin{tabular}{|c|c|c|c|c|}
\hline BDENF & LILACS & PubMed & SciELO & CINAHL \\
\hline $\mathbf{n}=\mathbf{3 0 3}$ & $\mathbf{n}=\mathbf{8 5 5}$ & $\mathbf{n}=\mathbf{4 . 4 2 9}$ & $\mathbf{n}=\mathbf{2 8}$ & $\mathbf{n}=\mathbf{1 . 0 6 3}$ \\
\hline
\end{tabular}
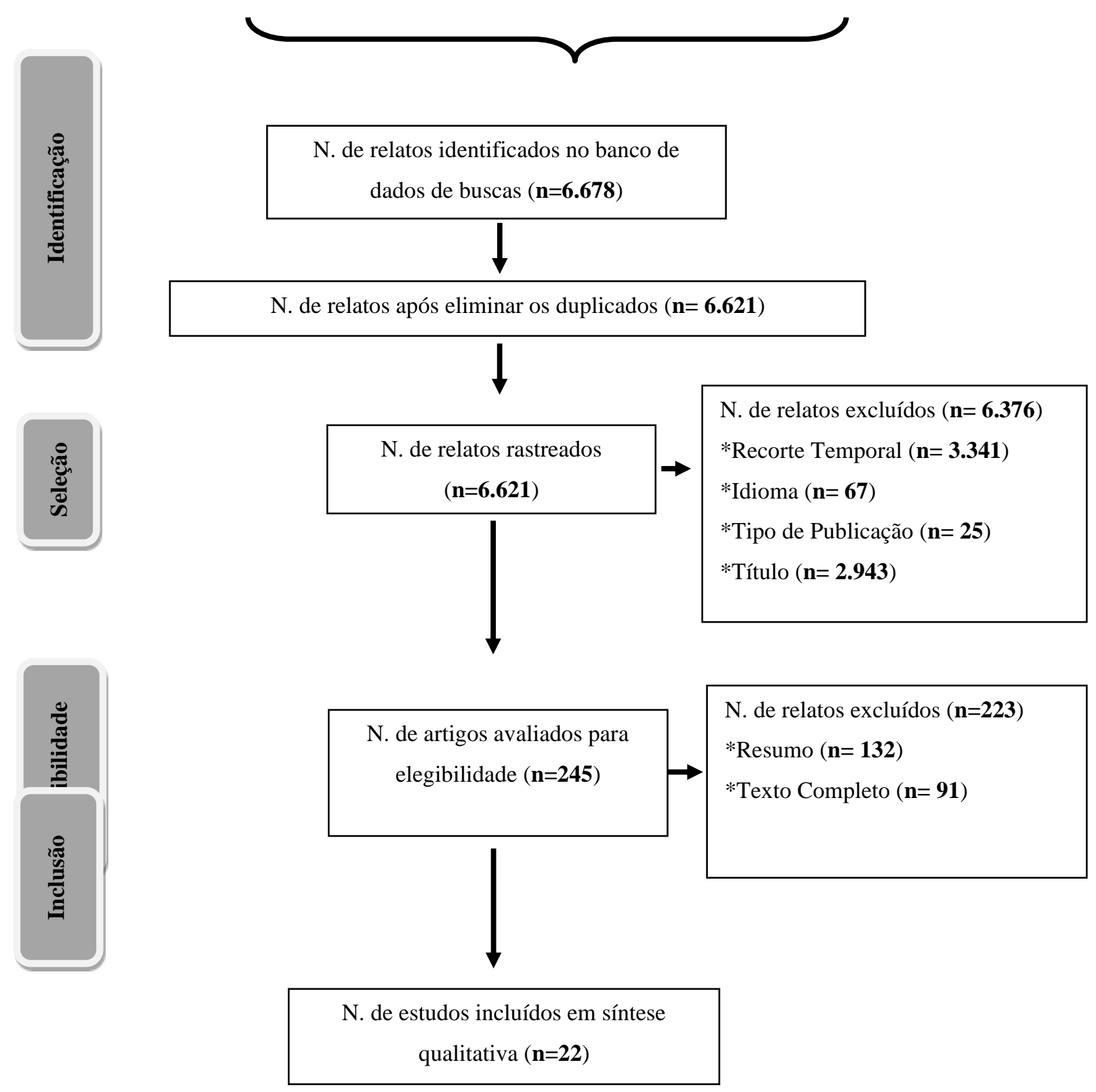

Fonte: PRISMA (Page et al., 2021). 
A Figura 1 apresenta a distribuição dos artigos por base de dados, cuja predominância em publicação foi a PubMed $(66,3 \%)$, seguida pela CINAHL $(15,9 \%)$.

O Quadro 2 compila algumas informações extraídas da amostra $(n=22)$ e revela que a maioria dos artigos teve os Estados Unidos como país de publicação, seguido pelo Brasil e China.

Quadro 2 - Apresentação dos artigos por autor, ano, país, periódico, método, objetivo e nível de evidência. Aracaju, SE, Brasil, 2021

\begin{tabular}{|c|c|c|c|c|c|}
\hline Código & Autor, Ano, País & Periódico & Método & Objetivo & $\begin{array}{l}\text { Nível de } \\
\text { Evidência }\end{array}$ \\
\hline A1 & $\begin{array}{l}\text { Aguiar, Pereira \& } \\
\text { Pinto } \\
\text { (2018) } \\
\text { Brasil }\end{array}$ & Rev. Eletr. Enf & $\begin{array}{l}\text { Estudo descritivo analítico } \\
\text { de caráter quantitativo }\end{array}$ & $\begin{array}{l}\text { Identificar os fatores que influenciam na } \\
\text { reconstrução do trânsito intestinal em } \\
\text { pessoas com estoma provisório. }\end{array}$ & VI \\
\hline A2 & $\begin{array}{c}\text { Costa, et al. (2017) } \\
\text { Brasil }\end{array}$ & $\begin{array}{l}\text { Rev Enferm Atual } \\
\text { In Derme }\end{array}$ & $\begin{array}{l}\text { Estudo transversal, } \\
\text { retrospectivo e descritivo }\end{array}$ & 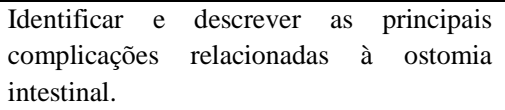 & VI \\
\hline A3 & $\begin{array}{l}\text { Ilyas, et al. (2018) } \\
\text { Estados Unidos }\end{array}$ & $\begin{array}{l}\text { J Wound Ostomy } \\
\text { Continence Nurs }\end{array}$ & Estudo Multicêntrico & $\begin{array}{l}\text { Demonstrar os resultados clínicos de } \\
\text { pacientes com ostomias temporárias em três } \\
\text { hospitais da Veterans } \\
\text { Administration. }\end{array}$ & $\mathrm{V}$ \\
\hline A4 & $\begin{array}{l}\text { Zhang, et al. (2019) } \\
\text { China }\end{array}$ & J Clin Nurs & $\begin{array}{l}\text { Estudo descritivo, } \\
\text { transversal }\end{array}$ & $\begin{array}{l}\text { Investigar a relação entre adaptação } \\
\text { psicossocial e qualidade de vida de } \\
\text { pacientes ostomizados. }\end{array}$ & VI \\
\hline A5 & $\begin{array}{l}\text { Altschuler, et al. } \\
\qquad(2018) \\
\text { Estados Unidos }\end{array}$ & $\begin{array}{l}\text { Support Care } \\
\text { Cancer }\end{array}$ & Estudo descritivo & $\begin{array}{l}\text { Discutir como a mutualidade pode afetar os } \\
\text { cuidados da ostomia a longo prazo. }\end{array}$ & VI \\
\hline A6 & $\begin{array}{l}\text { Krouse, et al. } \\
\qquad(2016) \\
\text { Estados Unidos }\end{array}$ & Psychooncology & Estudo piloto longitudinal & $\begin{array}{l}\text { Desenvolver um programa de autocuidado } \\
\text { para melhorar a qualidade de vida em } \\
\text { ostomizados. }\end{array}$ & IV \\
\hline A7 & $\begin{array}{l}\text { Millard, Cooper \& } \\
\text { Boyle (2020) } \\
\text { Estados Unidos }\end{array}$ & $\begin{array}{l}\text { Home Healthc } \\
\text { Now }\end{array}$ & $\begin{array}{l}\text { Estudo piloto } \\
\text { longitudinal }\end{array}$ & $\begin{array}{l}\text { Melhorar a qualidade de vida de pacientes } \\
\text { com ostomia, através de uma intervenção } \\
\text { baseada na educação do enfermeiro e do } \\
\text { paciente. }\end{array}$ & IV \\
\hline A8 & $\begin{array}{c}\text { Seo (2019) } \\
\text { Coreia do Sul }\end{array}$ & Int Wound J & Ensaio clínico controlado & $\begin{array}{l}\text { Avaliar os efeitos da educação de reforço } \\
\text { para gerenciamento da ostomia. }\end{array}$ & II \\
\hline A9 & $\begin{array}{l}\text { Xia }(2020) \\
\text { China }\end{array}$ & J Cancer Educ & $\begin{array}{c}\text { Ensaio clínico } \\
\text { randomizado simples-cego }\end{array}$ & $\begin{array}{l}\text { Examinar os efeitos do modelo de cuidado } \\
\text { contínuo de integração hospital-família } \\
\text { baseado nas informações dos pacientes. }\end{array}$ & II \\
\hline Código & Autor, Ano, País & Periódico & Método & Objetivo & $\begin{array}{l}\text { Nível de } \\
\text { Evidência }\end{array}$ \\
\hline A10 & $\begin{array}{c}\text { Sarah, Navipour \& } \\
\text { Mohammadi (2017) } \\
\text { Irã }\end{array}$ & World J Surg & Pesquisa qualitativa & $\begin{array}{l}\text { Identificar o principal problema dos } \\
\text { iranianos estomizados sobre o autocuidado. }\end{array}$ & VI \\
\hline A11 & $\begin{array}{c}\text { Karabulut, Dinç \& } \\
\text { Karadag (2014) } \\
\text { Turquia } \\
\end{array}$ & J Clin Nurs & Estudo quase experimental & $\begin{array}{l}\text { Investigar os efeitos de um método de } \\
\text { interação grupal no ajustamento social de } \\
\text { indivíduos ostomizados. }\end{array}$ & IV \\
\hline A12 & $\begin{array}{c}\text { Kerr (2015) } \\
\text { Estados Unidos }\end{array}$ & J Nurs Educ & Pesquisa qualitativa & $\begin{array}{l}\text { Investigar os benefícios potenciais de uma } \\
\text { simulação imersiva sobre o impacto da } \\
\text { ostomia. }\end{array}$ & VI \\
\hline A13 & $\begin{array}{l}\text { Pouresmail, et al. } \\
\text { (2019) } \\
\text { Irã } \\
\end{array}$ & $\begin{array}{l}\text { Wound Manag } \\
\text { Prev }\end{array}$ & $\begin{array}{c}\text { Ensaio clínico } \\
\text { randomizado simples-cego }\end{array}$ & $\begin{array}{l}\text { Determinar os efeitos da simulação no } \\
\text { treinamento de autocuidado com ostomia } \\
\text { sobre a autoeficácia e o ajuste. }\end{array}$ & II \\
\hline A14 & $\begin{array}{c}\text { Braumann, et al. } \\
\text { (2016) } \\
\text { Alemanha } \\
\end{array}$ & $\begin{array}{l}\text { Langenbecks } \\
\text { Arch Surg }\end{array}$ & $\begin{array}{l}\text { Estudo observacional do } \\
\text { tipo Survey }\end{array}$ & $\begin{array}{l}\text { Descrever a situação real dos pacientes } \\
\text { ostomizados e identificar os parâmetros que } \\
\text { influenciam a qualidade de vida. }\end{array}$ & VI \\
\hline A15 & $\begin{array}{l}\text { Coca, et al. (2015) } \\
\text { Espanha }\end{array}$ & $\begin{array}{l}\text { J Wound Ostomy } \\
\text { Continence Nurs }\end{array}$ & $\begin{array}{c}\text { Estudo multicêntrico, } \\
\text { quase experimental, } \\
\text { prospectivo e longitudinal. }\end{array}$ & $\begin{array}{l}\text { Comparar a qualidade de vida entre um } \\
\text { grupo de pacientes atendidos por } \\
\text { enfermeiras da WOCN* e generalistas. }\end{array}$ & IV \\
\hline A16 & $\begin{array}{l}\text { Zhang, et al. (2013) } \\
\text { China }\end{array}$ & Cancer Nurs & $\begin{array}{l}\text { Ensaio clínico } \\
\text { randomizado }\end{array}$ & $\begin{array}{l}\text { Avaliar o efeito do acompanhamento } \\
\text { telefônico da estomaterapeuta nos níveis de }\end{array}$ & II \\
\hline
\end{tabular}


Research, Society and Development, v. 11, n. 2, e11211225496, 2022

(CC BY 4.0) | ISSN 2525-3409 | DOI: http://dx.doi.org/10.33448/rsd-v11i2.25496

\begin{tabular}{|c|c|c|c|c|c|}
\hline & & & & $\begin{array}{l}\text { ajuste de pacientes com colostomia pós- } \\
\text { alta. }\end{array}$ & \\
\hline A17 & $\begin{array}{l}\text { Thorpe, McArthur } \\
\text { \& Richardson } \\
\text { (2014) } \\
\text { Reino Unido }\end{array}$ & $\begin{array}{c}\text { Int } \\
\text { J Nurs Stud }\end{array}$ & $\begin{array}{l}\text { Estudo fenomenológico- } \\
\text { existencial }\end{array}$ & $\begin{array}{l}\text { Explorar a experiência individual de } \\
\text { conviver com um novo estoma e as } \\
\text { interações com a saúde ao longo do tempo. }\end{array}$ & VI \\
\hline A18 & $\begin{array}{l}\text { Nafees, et al. (2018) } \\
\text { Reino Unido }\end{array}$ & $\begin{array}{c}\text { Health } \\
\text { Qual Life } \\
\text { Outcomes }\end{array}$ & Estudo metodológico & $\begin{array}{l}\text { Validar uma ferramenta para medir o } \\
\text { impacto do vazamento em pessoas que } \\
\text { utilizam dispositivos para estomas. }\end{array}$ & VI \\
\hline Código & Autor, Ano, País & Periódico & Método & Objetivo & $\begin{array}{l}\text { Nível de } \\
\text { Evidência }\end{array}$ \\
\hline A19 & $\begin{array}{l}\text { Pereira, et al. (2012) } \\
\text { Brasil }\end{array}$ & $\begin{array}{l}\text { Rev. Latino-Am. } \\
\text { Enfermagem }\end{array}$ & Estudo transversal & $\begin{array}{l}\text { Identificar os fatores sociodemográficos e } \\
\text { clínicos dos pacientes com estoma intestinal } \\
\text { definitivo. }\end{array}$ & VI \\
\hline A20 & $\begin{array}{l}\text { Corvese, et al. } \\
(2020) \\
\text { Itália }\end{array}$ & Br J Nurs & Pesquisa Survey & $\begin{array}{l}\text { Identificar as características } \\
\text { sociodemográficas e clínicas de pacientes } \\
\text { italianos ostomizados. }\end{array}$ & VI \\
\hline A21 & $\begin{array}{l}\text { Cengiz \& Bahar } \\
\text { (2017) } \\
\text { Turquia }\end{array}$ & $\begin{array}{l}\text { J Wound Ostomy } \\
\text { Continence Nurs }\end{array}$ & $\begin{array}{l}\text { Estudo fenomenológico- } \\
\text { existencial }\end{array}$ & $\begin{array}{l}\text { Identificar as barreiras percebidas para a } \\
\text { adaptação à vida com ostomia fecal com } \\
\text { base no Modelo de Crenças de Saúde. }\end{array}$ & VI \\
\hline A22 & $\begin{array}{l}\text { Coelho, et al. } \\
\text { (2015) } \\
\text { Brasil }\end{array}$ & $\begin{array}{l}\text { Rev enferm UFPE } \\
\text { on line }\end{array}$ & $\begin{array}{l}\text { Estudo exploratório, } \\
\text { transversal, com } \\
\text { abordagem quantitativa } \\
\end{array}$ & $\begin{array}{l}\text { Analisar o autocuidado de pacientes } \\
\text { colostomizados, a pele periestoma e o } \\
\text { dispositivo coletor. }\end{array}$ & VI \\
\hline
\end{tabular}

WOCN - Wound, Ostomy and Continence Nurses Society. Fonte: Elaborado pelos autores (2021) baseado no instrumento de Ursi e Galvão (2006).

No que se refere aos objetivos da amostra ( $\mathrm{n}=22)$, os artigos A6, A7, A8, A9, A11, A13 e A16 abordaram algum tipo de programa de educação ou intervenção voltado para o gerenciamento do autocuidado, além dos cuidados de enfermagem. $\mathrm{O}$ artigo A18 trata de uma validação psicométrica, a qual objetivou o desenvolvimento de uma nova ferramenta de medição para entender o impacto do vazamento da ostomia. Os artigos A17 e A21 revelaram as percepções do indivíduo sobre o estoma sob a perspectiva fenomenológica. Os demais artigos se dedicaram a discriminar os fatores de risco, fatores que afetam a reversão do estoma, complicações pós-operatórias (hérnia, prolapso) relacionadas ao estoma, qualidade de vida, mutualidade, interação social e familiar.

O Quadro 3 reúne informações sobre a seleção da amostra, os principais resultados e os cuidados de enfermagem. Quanto à seleção da amostra $(n=22)$, os estudos foram categorizados em: intencional $(54,5 \%)$, randômica $(27,2 \%)$, conveniência $(18,1 \%)$.

Quadro 3 - Caracterização dos artigos por seleção da amostra, principais resultados e cuidados de enfermagem. Aracaju, SE, Brasil, 2021.

\begin{tabular}{|c|c|c|c|}
\hline Código & $\begin{array}{l}\text { Seleção da } \\
\text { Amostra }\end{array}$ & Principais resultados & Cuidados de enfermagem \\
\hline A1 & Conveniência & $\begin{array}{l}\text { Associação significativa entre os motivos da demora na reconstrução do } \\
\text { trânsito intestinal e o tipo de patologia (abdome agudo, neoplasias) } \\
(\mathrm{p}=0,004) \text {. }\end{array}$ & $\begin{array}{l}\text { Estomaterapeuta deve subsidiar a estruturação, } \\
\text { implantação e avaliação de um protocolo de } \\
\text { cuidado em rede. }\end{array}$ \\
\hline Código & $\begin{array}{l}\text { Seleção da } \\
\text { Amostra }\end{array}$ & Principais resultados & Cuidados de enfermagem \\
\hline $\mathrm{A} 2$ & Conveniência & $\begin{array}{l}\text { O prolapso se deu } 25 \% \text { de forma precoce e } 75 \% \text { tardia. Em relação à } \\
\text { expectativa de tempo do estoma, } 45,4 \% \text { das colostomias foram } \\
\text { confeccionadas de forma terminal. }\end{array}$ & $\begin{array}{l}\text { Dispositivo coletor de duas peças para melhor } \\
\text { adaptar a alça intestinal; Redução manual da } \\
\text { alça intestinal. }\end{array}$ \\
\hline A3 & Intencional & $\begin{array}{l}\text { Houve reversão do estoma em } 50 \% \text { dos pacientes, com duração mediana } \\
\text { de } 9 \text { meses entre a formação e reversão. }\end{array}$ & $\begin{array}{l}\text { Aconselhamento do paciente quanto às } \\
\text { complicações pós-operatórias e orientação } \\
\text { quanto à reversão ou não dos estomas } \\
\text { temporários. }\end{array}$ \\
\hline A4 & Conveniência & $\begin{array}{l}20,9 \% \text { desenvolveu complicação periestoma e } 72,2 \% \text { apresentou } \\
\text { vazamento durante os últimos } 3 \text { meses. }\end{array}$ & $\begin{array}{l}\text { Educação pré-operatória (localização do } \\
\text { estoma antes da cirurgia, tipo de estoma, tipo de } \\
\text { bolsa de colostomia, complicações }\end{array}$ \\
\hline
\end{tabular}


Research, Society and Development, v. 11, n. 2, e11211225496, 2022

(CC BY 4.0) | ISSN 2525-3409 | DOI: http://dx.doi.org/10.33448/rsd-v11i2.25496

\begin{tabular}{|c|c|c|c|}
\hline & & & $\begin{array}{l}\text { periestomais, habilidade de autocuidado do } \\
\text { estoma, comunicação com a equipe médica). }\end{array}$ \\
\hline A5 & Intencional & $\begin{array}{l}58 \% \text { precisava de algum tipo de suporte para realizar as atividades de } \\
\text { vida diária. }\end{array}$ & $\begin{array}{l}\text { Pacotes diários de cuidados para ostomia } \\
\text { contendo lenços umedecidos e assistência para } \\
\text { ajudar a colocar o dispositivo no paciente. }\end{array}$ \\
\hline A6 & Intencional & $\begin{array}{l}\text { Autoeficácia para o manejo da ostomia: alfa pré-sessão }(0,96) \text {; pós- } \\
\text { intervenção }(0,90) \text { e acompanhamento }(0,86) \text {; Em relação ao PAM, } \\
\text { foram evidenciados o seguintes resultados: } 0,70 ; 0,87 \text {; e } 0,94 \text {. }\end{array}$ & $\begin{array}{l}\text { Programa de autogestão sistemático baseado } \\
\text { nas necessidades dos ostomizados. }\end{array}$ \\
\hline A7 & Intencional & $\begin{array}{l}\text { O número de semanas diminuiu em média 1,5 após a intervenção, e todos } \\
\text { os quatro pacientes precisaram de apenas um atendimento domiciliar. }\end{array}$ & $\begin{array}{l}\text { Programa de treinamento de autogestão de } \\
\text { ostomia. }\end{array}$ \\
\hline A8 & Randômica & $\begin{array}{l}\text { Os efeitos da frequência do OMRE sobre a capacidade de trocar o } \\
\text { aparelho do estoma foram testados, e os resultados mostraram } \\
\text { pontuações significativamente mais altas para os grupos experimentais } 1 \\
\text { e } 2 \text { do que para o grupo de controle }(\mathrm{p}<0,001) \text {. }\end{array}$ & OMRE. \\
\hline A9 & Randômica & $\begin{array}{l}\text { Este modelo fortaleceu significativamente a autoeficácia e a confiança } \\
\text { dos pacientes, o que diminuiu as complicações da colostomia. }\end{array}$ & $\begin{array}{l}\text { Modelo de cuidados de enfermagem contínuo } \\
\text { baseado em informações em pacientes com } \\
\text { câncer retal submetidos à cirurgia de Miles. }\end{array}$ \\
\hline Código & $\begin{array}{c}\text { Seleção da } \\
\text { Amostra }\end{array}$ & Principais resultados & Cuidados de enfermagem \\
\hline A10 & Intencional & $\begin{array}{l}\text { Pacientes relataram vazamento, odor e ruído e uma preocupação em } \\
\text { alcançar o gerenciamento eficaz. Evidenciou-se uma ligação entre o fator } \\
\text { tempo e a capacidade de gerenciar gases e vazamento. }\end{array}$ & $\begin{array}{l}\text { Estomaterapeuta auxilia na adaptação } \\
\text { fisiológica e psicossocial dos pacientes, } \\
\text { educando-os sobre como administrar e lidar } \\
\text { com uma ostomia intestinal. }\end{array}$ \\
\hline A11 & Intencional & $\begin{array}{l}\text { Membros do grupo experimental tinham conhecimento suficiente sobre } \\
\text { cuidados com o estoma demonstraram valores de ajuste mais altos }(\mathrm{p}< \\
0 \text { 05). }\end{array}$ & $\begin{array}{l}\text { Método de interação de grupo planejado na } \\
\text { adaptação social de indivíduos com estoma } \\
\text { intestinal; Reabilitação com o estomaterapeuta. }\end{array}$ \\
\hline A12 & Intencional & $\begin{array}{l}\text { Identificou-se aborrecimentos, dificuldades para realizar as atividades de } \\
\text { vida diária, inclusive a posição prona, medo de vazamento. }\end{array}$ & $\begin{array}{l}\text { Demarcação pré-operatória; Visita de um } \\
\text { enfermeiro WOCN. }\end{array}$ \\
\hline A13 & Randômica & $\begin{array}{l}\text { Melhora na autoeficácia em ambos os grupos desde o início até a visita } \\
\text { de acompanhamento final }(\mathrm{p}<0,0001) \text {. }\end{array}$ & $\begin{array}{l}\text { Simulação de treinamento para autocuidado } \\
\text { com ostomia sobre a autoeficácia e ajuste. }\end{array}$ \\
\hline A14 & Randômica & $\begin{array}{l}\text { O manejo de complicações foi melhor conduzido em pacientes com } \\
\text { ileostomia }(p<0,01) \text {. }\end{array}$ & $\begin{array}{l}\text { Demarcação pré-operatória; Avaliação das } \\
\text { taxas de complicações e acompanhamento } \\
\text { contínuo pelo enfermeiro WOCN. }\end{array}$ \\
\hline A15 & Randômica & $\begin{array}{l}\text { Grupo } 1 \text { era mais propenso à demarcação do estoma em relação ao grupo } \\
2(p>0,001) . O \text { grupo } 1 \text { relatou se sentir mais seguro em limpar seu } \\
\text { estoma }(p=0,005) .\end{array}$ & $\begin{array}{l}\text { Demarcação pré-operatória; Gerenciamento da } \\
\text { ostomia pela enfermeira WOCN. }\end{array}$ \\
\hline A16 & Randômica & $\begin{array}{l}\text { Em } 1 \text { mês (intervenção } 1,44 \text { vs controle } 2,12, \mathrm{t}=4,85, \mathrm{P}=.000 \text { ) e } 3 \text { meses } \\
\text { (intervenção } 1,45 \text { vs controle } 2,04, \mathrm{t}=4,45, \mathrm{P}=.000 \text { ) após a alta, o grupo } \\
\text { intervenção teve uma satisfação significativamente maior com o } \\
\text { atendimento. }\end{array}$ & $\begin{array}{l}\text { Acompanhamento por telefone da enfermeira } \\
\text { WOCN. }\end{array}$ \\
\hline A17 & Intencional & $\begin{array}{l}\text { A educação pré-operatória foi valorizada por quase todos, mesmo } \\
\text { aqueles que não a receberam devido à cirurgia de emergência. }\end{array}$ & $\begin{array}{l}\text { Educação pré-operatória; reabilitação com a } \\
\text { enfermeira WOCN; assistência com a } \\
\text { enfermeira generalista. }\end{array}$ \\
\hline A18 & Intencional & $\begin{array}{l}\text { Alta correlação entre os domínios da ferramenta de vazamento e o } \\
\text { domínio Impacto emocional quando comparado com SF-36, bem-estar } \\
\text { emocional e domínio de confiança do CoH-QoL-OQ }(\mathrm{p}<0,001) \text {. }\end{array}$ & $\begin{array}{l}\text { Desenvolvimento de um instrumento para } \\
\text { avaliar a natureza dos vazamentos das } \\
\text { ostomias. }\end{array}$ \\
\hline Código & $\begin{array}{l}\text { Seleção da } \\
\text { Amostra }\end{array}$ & Principais resultados & Cuidados de enfermagem \\
\hline A19 & Intencional & $\begin{array}{l}\text { Em } 60 \% \text { dos casos não foi demarcado. O domínio físico e psicológico } \\
\text { foi significativamente afetado ( } \mathrm{p}=0,023 \text { ) nos pacientes que não foram } \\
\text { orientados antes da cirurgia sobre a confecção do estoma. }\end{array}$ & Demarcação pré-operatória. \\
\hline A20 & Intencional & $\begin{array}{l}\text { A escolha do dispositivo de ostomia foi feita de forma independente por } \\
24,9 \% \text { dos pacientes; em } 61,9 \% \text { dos casos foi escolhido pela enfermeira } \\
\text { WOCN. }\end{array}$ & $\begin{array}{l}\text { Dispositivos para manejo de complicações; } \\
\text { Orientações da enfermeira WOCN. }\end{array}$ \\
\hline A21 & Intencional & $\begin{array}{l}\text { O aconselhamento conduzido por enfermeiras por meio de ligações } \\
\text { telefônicas e visitas domiciliares em intervalos de } 2 \text { a } 4 \text { semanas. }\end{array}$ & $\begin{array}{l}\text { Educação pré-operatória; Acompanhamento } \\
\text { pela enfermeira WOCN por meio telefônico; } \\
\text { Visita domiciliar; Reforço pós-alta. }\end{array}$ \\
\hline A22 & Conveniência & $\begin{array}{l}55,7 \% \text { pessoas afirmaram realizar a troca do dispositivo dentro do prazo } \\
\text { de três a quatro dias. O enfermeiro foi citado por } 65,3 \% \text { dos pacientes } \\
\text { como profissional que proferiu as primeiras orientaçôes. }\end{array}$ & $\begin{array}{l}\text { Cuidados relativos ao estoma, à pele } \\
\text { periestoma e ao dispositivo coletor. }\end{array}$ \\
\hline
\end{tabular}

PAM - Patient Activation Measure; OMRE - Ostomy Management Reinforcement Education; WOCN - Wound, Ostomy and Continence Nurses Society; SF-36 - Medical Outcomes Study 36-Item Short-Form Health Survey; CoH-QoL-OQ - City of Hope Quality of LifeOstomy Questionnaire. Fonte: Elaborado pelos autores (2021) baseado no instrumento de Ursi e Galvão (2006). 
Em se tratando dos cuidados de enfermagem, os estudos A1, A3, A4, A10, A11, A12, A14, A15, A16, A17, A19, A20 e A21 enfatizaram alguns cuidados relacionados à prevenção do prolapso, tais como: educação pré-operatória, demarcação préoperatória do estoma, dispositivos para manejo de complicações, orientação pós-operatória, visita domiciliar e acompanhamento via telefônico realizado pelo estomaterapeuta. Os demais estudos se concentraram em discutir programas de gerenciamento da ostomia, modelos de cuidados de enfermagem e outros aspectos relacionados à adaptação do indivíduo ao estoma (Quadro 3).

\section{Discussão}

A presente revisão evidenciou os Estados Unidos no topo de publicações, o que se justifica pelo maior número de estudos rastreados pela PubMed, bem como, se deve ao fato da WOCN - sociedade científica de enfermagem dedicada ao avanço da prática e prestação de cuidados especializados para indivíduos com necessidades de cuidados de feridas, ostomia e continência -, estar localizada em New Jersey e ser uma referência internacional que enfatiza a importância do enfermeiro WOCN na reabilitação desse público. Atrelado a isso, os EUA possuem 1 milhão de pessoas vivendo com estoma, e aproximadamente 120.000 estomas são confeccionados anualmente no país (McGee, 2016).

Dentre os estudos selecionados nesta revisão, quatro deles (A12, A14, A15 e A19) demonstraram que a demarcação pré-operatória é uma importante estratégia de cuidado para prevenção do prolapso em ostomias intestinais. Alinhada à demarcação, faz-se necessário o emprego de técnica cirúrgica meticulosa, a fim de reduzir a taxa dessa complicação (Hsu et al., 2020). Corroborando a eficácia de tal estratégia, pesquisa realizada com 364 pacientes evidenciou que $70 \%$ não foi demarcado no pré-operatório e, na análise multivariada, a demarcação foi independentemente associada à redução das taxas de complicações (Arolfo et al., 2018).

A garantia de um sítio cirúrgico adequado ao estoma favorece a aderência do equipamento e a visualização pelo paciente, constituindo-se uma estratégia preventiva de complicações como o prolapso e a hérnia paraestomal (Murken \& Bleier, 2019). Outros autores convergem, nesse mesmo ponto, sobre o fato da maioria das complicações relacionadas ao estoma pode ser evitada mediante um planejamento do local específico do estoma no abdome e da técnica cirúrgica realizada, principalmente nas colostomias e ileostomias definitivas (De Araújo et al., 2021).

Nessa perspectiva, foi conduzida uma revisão sistemática com metanálise, na qual foram incluídos 2.109 participantes, comparando dois grupos de pacientes: os que se submeteram à marcação pré-operatória do estoma e aqueles que não experimentaram o procedimento. Evidenciou-se que a marcação foi associada à redução do estoma e complicações periestomais em todos os tipos de ostomia ( $\mathrm{p}<0,001)$. Além disso, indivíduos submetidos a ostomias intestinais apresentaram menos complicações $(\mathrm{p}<0,001)$ em relação àqueles que não tiveram o estoma demarcado (Hsu et al., 2020).

Neste tocante, a demarcação do estoma é recomendada em várias diretrizes, incluindo a americana (Hendren et al., 2015) e diretrizes alemãs (Krebsgesellschaft \& Deutsche, 2019). Contudo, não há consenso sobre como marcar e quem deve fazer a marcação. Entretanto, autores recomendam que o procedimento seja efetuado pelo cirurgião líder ou por um membro experiente da equipe cirúrgica ou até mesmo por um estomaterapeuta (Wassemann \& McGee, 2017).

Os artigos (A4, A17 e A21) revelaram que a educação pré-operatória constitui-se uma tecnologia com o potencial de instrumentalizar o paciente acerca da localização do estoma previamente à cirurgia, qual tipo será confeccionado e como minimizar as complicações pós-operatórias. Nesta perspectiva, estudos prévios indicaram que a consulta pré-operatória inclui a demarcação com a fixação de uma placa de base de teste, além disso, os pacientes devem receber treinamento da enfermeira WOCN, a fim de que sejam capazes de cuidar de sua ostomia com confiança e segurança após a alta hospitalar (Harris, Kelly \& Parise, 2020; Bulkley et al., 2018).

Nesse contexto, ressalta-se a importância da educação pré-operatória no acompanhamento realizado por uma enfermeira especialista com foco no autocuidado da ostomia e/ou assistência do cuidador. Uma possível explicação seria que os participantes 
deste estudo foram regularmente vistos por estomaterapeutas que os orientaram sobre como reconhecer e tratar as complicações relacionadas ao estoma (Wen et al., 2019; Carlsson et al., 2016).

Os estudos (A16 e A21) enfatizaram o acompanhamento telefônico educacional e de suporte pós-alta realizado pela enfermeira WOCN. Estudo prévio destaca o fato de que esse acompanhamento influencia o nível de ajuste do paciente, à medida que esse aprende a lidar com os problemas do autocuidado, e passa a dominar suas habilidades e resolver questões relacionadas às suas atividades de vida diária, incluindo a prevenção de complicações como o prolapso (Hamidi, Moeini \& Yousefi, 2018). Isso é ratificado por uma metanálise de 16 estudos, que avaliou 2.912 pacientes oncológicos, os quais foram submetidos a intervenções telefônicas, e notabilizou-se pela melhora no autocuidado e na qualidade de vida (Suh \& Lee, 2017).

Os estudos (A1, A10, A14, e A17) contribuíram acerca da reabilitação realizada pelo estomaterapeuta ou enfermeiro WOCN com vistas à redução das complicações pós-operatórias e melhoria na qualidade de vida. Neste sentido, diretrizes clínicas da WOCN apontam que a demarcação do estoma deve ser realizada por um clínico treinado para promover a independência do paciente nos cuidados com o estoma; utilizar um cinto de suporte de hérnia com um acessório de prolapso; determinar se um sistema de bolsa de uma peça pode minimizar o trauma ao estoma prolapsado (Wound, Ostomy and Continence Nurses Society, 2018).

A WOCN também recomenda que o especialista oriente o paciente ou cuidador quanto às técnicas para reduzir o estoma prolapsado; avaliação quanto a alterações de cor e procurar atendimento médico, se o estoma ficar escuro ou isquêmico, entre outras. Entretanto, essas diretrizes carecem de maiores estudos, tendo em vista que a WOCN é uma sociedade norte-americana, e suas evidências talvez não possam ser totalmente replicáveis em outros países, em virtude das diferenças regionais, clínicoepidemiológicas, bem como, a disponibilidade de dispositivos e adjuvantes para o gerenciamento do estoma (Wound, Ostomy and Continence Nurses Society, 2018).

No entanto, o estudo (A1) vinculou a função do estomaterapeuta à elaboração, implantação e avaliação de um protocolo de cuidado em rede. Além disso, a pesquisa também ressalta que essa atividade ainda é pouco disseminada no Brasil. Nesse contexto, estudo realizado na Espanha com 679 pacientes utilizando o protocolo Enhanced Recovery After Surgery (ERAS) em cirurgia colorretal demonstrou que a conformidade geral do protocolo ERAS (conjunto de medidas perioperatórias que visam reduzir o tempo de internação e as complicações pós-operatórias) na coorte pós-ERAS foi de $88 \%$, o que representa uma redução significativa das complicações e do tempo de internação (Ripollés-Melchor et al., 2018).

Outro estudo, que também utilizou a abordagem do protocolo ERAS, evidenciou que todos os pacientes são encorajados a manter um diário para registrar quanto comem e com que frequência estão ativos no pós-operatório. Todavia, a pesquisa revelou que os pacientes perdem o interesse em registrar suas atividades de reabilitação (por exemplo: comer e beber) ou em seguir as instruções relacionadas a essas atividades quando os profissionais de saúde não mostram interesse em ouvi-los, o que aponta a necessidade de acompanhamento do estomaterapeuta, inclusive, para orientar o paciente quanto à necessidade de reversão do estoma nos casos de colostomias temporárias (Wennström et al., 2020).

Os estudos (A6, A8, A9 e A13) evidenciaram a importância de programas de educação e gerenciamento do cuidado com a ostomia na minimização de complicações pós-operatórias e melhoria da qualidade de vida, com ênfase na obtenção de níveis mais elevados de autocuidado e autoeficácia. Diante disso, é válido ressaltar que o uso desses programas influenciam direta ou indiretamente no gerenciamento da ostomia e, consequentemente, na prevenção do prolapso, embora as evidências ainda suscitem de maiores esclarecimentos.

Destarte et al. (2016) ressaltam que um modelo de educação centrado no paciente, promovendo a autogestão e a colaboração entre pacientes e especialistas, alcança melhores resultados do que um programa didático em que os pacientes recebem passivamente informações padronizadas. 
Neste sentido, pesquisa sobre o impacto de um programa de educação associado à vigilância pós-operatória demonstrou que as visitas pré-operatória da enfermeira WOCN ocorreram com menos frequência, devido a ileostomia não ter feito parte do plano cirúrgico inicialmente (por exemplo, ressecção sigmóide para diverticulite) ou que a decisão de agendar uma cirurgia eletiva não permitiu tempo suficiente para uma consulta com a especialista (Grahn et al., 2019), o que evidencia claramente a necessidade do estomaterapeuta no pré-operatório, bem como, urge a necessidade de protocolos assistenciais que visem melhorar as taxas de complicações conforme demonstrado no estudo anterior sobre o protocolo ERAS (Ripollés-Melchor et al., 2018).

A presente pesquisa evidenciou limitações metodológicas importantes, pois, apesar da grande quantidade de trabalhos desenvolvidos a respeito da prevenção de prolapso em ostomias intestinais, poucos (A8, A9, A13 e A16) apresentaram um bom nível de evidência. Além disso, os resultados aqui demonstrados expressam apenas um retrato da literatura científica nesse tempo e espaço. Outrossim, a inclusão de cinco bases de dados, para a busca de estudos, bem como a delimitação do recorte temporal, pode não ter sido suficiente para exaurir a literatura científica a respeito da temática, o que pode ter levado à não inclusão de pesquisas elegíveis para compor esta revisão.

No entanto, esta revisão contribui para o gerenciamento do autocuidado em ostomias intestinais, ao abrir a possibilidade para desenvolver novas pesquisas sobre os cuidados relacionados à prevenção do prolapso e, quiçá, seja o ponto de partida para o desenvolvimento de metanálise para avaliar a eficácia entre a realização da demarcação pré-operatória e sua não realização. Além disso, cabe enfatizar que a educação pré-operatória e o acompanhamento telefônico pós-alta realizado por estomaterapeuta são cuidados relevantes para a prevenção do prolapso.

\section{Conclusão}

A demarcação cirúrgica prévia do estoma associada à educação pré-operatória e aos programas de educação e/ou intervenção voltados para o gerenciamento da ostomia no pós-operatório podem ser consideradas importantes tecnologias de cuidado capazes de prevenir o prolapso em ostomias intestinais. Todavia, a realização da demarcação ainda carece de evidências científicas mais robustas, tendo em vista que sua prática ainda não é efetivada, em larga escala, no âmbito internacional.

Os principais resultados do estudo trazem à tona a importância do desenvolvimento de modelos de cuidados e de programas de educação e de gerenciamento da ostomia voltados para a realidade brasileira, de modo que possam avaliar o impacto da educação e da demarcação pré-operatória na prevenção de complicações, como o prolapso. Neste sentido, os serviços de cirurgia poderiam incluir como rotina uma consulta pré-operatória do paciente com o estomaterapeuta para esclarecer as principais dúvidas sobre o procedimento cirúrgico e uma consulta e visita pós-operatória com esse especialista.

A partir dos achados do estudo, sugere-se o desenvolvimento de estudos que evidenciem a busca de tecnologias ou programas de gerenciamento da ostomia voltados à prevenção de prolapso em repositórios de patente, através da consulta à base de dados do Instituto Nacional de Propriedade Industrial (INPI), além de buscas na Patentscope vinculado a Word Intelectual Property Organization (WIPO) e mediante a busca no Espacenet da European Patent Office (EPO). Além disso, os resultados fornecem aos autores e à comunidade científica evidências para elaborar softwares e/ou desenvolver algoritmos de prevenção de prolapso em ostomias intestinais.

\section{Referências}

Aguiar, J. C., Pereira, A. P. dos S., \& Pinto, M. H. (2018). Reconstrução de trânsito intestinal: fatores que influenciam a realização. Revista Eletrônica De Enfermagem, 20(32), 1-10.

Almeida, A. O. D., Dantas, S. R. P. E., Paula, M. A. B. D., Silva, J. L. G., Franck, E. M., \& Oliveira-Kumakura, A. R. D. S. (2021). Construção, validação e aplicação de cenários de simulação clínica para avaliação de especialistas em estomaterapia. Revista Brasileira de Enfermagem, 74(1).

Altschuler, A., Liljestrand, P., Grant, M., Hornbrook, M. C., Krouse, R. S., \& McMullen, C. K. (2018). Caregiving and mutuality among long-term colorectal cancer survivors with ostomies: qualitative study. Supportive Care in Cancer, 26(2), 529-537. 
Ambe, P. C., Kurz, N. R., Nitschke, C., Odeh, S. F., Möslein, G., \& Zirngibl, H. (2018). Intestinal ostomy: classification, indications, ostomy care and complication management. Deutsches Ärzteblatt International, 115(11), 182.

Arolfo, S., Borgiotto, C., Bosio, G., Mistrangelo, M., Allaix, M. E., \& Morino, M. (2018). Preoperative stoma site marking: a simple practice to reduce stomarelated complications. Techniques in coloproctology, 22(9), 683-687.

Braumann, C., Müller, V., Knies, M., Aufmesser, B., Schwenk, W., \& Koplin, G. (2016). Quality of life and need for care in patients with an ostomy: a survey of 2647 patients of the Berlin OStomy-Study (BOSS). Langenbeck's archives of surgery, 401(8), 1191-1201.

Bulkley, J. E., McMullen, C. K., Grant, M., Wendel, C., Hornbrook, M. C., \& Krouse, R. S. (2018). Ongoing ostomy self-care challenges of long-term rectal cancer survivors. Supportive care in cancer, 26(11), 3933-3939.

Byfield, D. (2020). The Lived Experiences of Persons With Ostomies Attending a Support Group: A Qualitative Study. Journal of Wound, Ostomy and Continence Nursing, 47(5), 489-495.

Carlsson, E., Fingren, J., Hallén, A. M., Petersén, C., \& Lindholm, E. (2016). The prevalence of ostomy-related complications 1 year after ostomy surgery: A prospective, descriptive, clinical study. Ostomy/wound management, 62(10), 34-48.

Cengiz, B., \& Bahar, Z. (2017). Perceived barriers and home care needs when adapting to a fecal ostomy. Journal of Wound, Ostomy and Continence Nursing, 44(1), 63-68.

Coca, C., Fernández de Larrinoa, I., Serrano, R., \& García-Llana, H. (2015). The impact of specialty practice nursing care on health-related quality of life in persons with ostomies. Journal of Wound, Ostomy and Continence Nursing, 42(3), 257-263.

Coelho, A. M. S., de Oliveira, C. G., Bezerra, S. T. F., Almeida, A. N. S., Cabral, R. L., \& Coelho, M. M. F. (2015). Self care of patients with colostomy, peristomal skin and collecting bag. J Nurs UFPE on line, 9, 9528-34.

Corvese, F., Giordano, V., Alvaro, R., Vellone, E., \& Villa, G. (2020). Sociodemographic characteristics and self-care management knowledge of patients with an ostomy. British Journal of Nursing, 29(22), S20-S26.

Costa, J. M. da, Ramos, R. de S., Santos, M. M. dos, Silva, D. F. da, Gomes, T. da S., \& Batista, R. Q. (2017). Complicações do estoma intestinal em pacientes em pós- operatório de ressecção de tumores de reto. Revista Enfermagem Atual In Derme, 34-42.

De Araújo, A. C., Menezes A. M., Ramirez A. C. F. O., Kotovicz B. B. de M., Ueda C. C., Fernandes Ítalo R. de Q., Lopes J. R., Rodrigues L. P., Dourado M. V. F., \& Corrêa W. P. (2021). Principais complicações da reconstrução do trato intestinal após colostomia à Hartmann. Revista Eletrônica Acervo Saúde, 13(3), e6594.

De Melo, G. D. N., De Meireles, D. S., de Araújo, C. S., \& dos Santos, M. S. (2021). Autoimagem de mulheres portadoras de colostomia e os cuidados dermatológicos periestoma: revisão integrativa. Brazilian Journal of Health Review, 4(1), 991-1001.

Duluklu, B., \& Çelik, S. Ş. (2019). Effects of lavender essential oil for colorectal cancer patients with permanent colostomy on elimination of odor, quality of life, and ostomy adjustment: A randomized controlled trial. European Journal of Oncology Nursing, 42, 90-96.

Estrada, D. M. L., Benghi, L. M., \& Kotze, P. G. (2021). Practical insights into stomas in inflammatory bowel disease: what every healthcare provider needs to know. Current Opinion in Gastroenterology, 37(4), 320-327.

Giordano, V., Nicolotti, M., Corvese, F., Vellone, E., Alvaro, R., \& Villa, G. (2020). Describing self-care and its associated variables in ostomy patients. Journal of Advanced Nursing, 76(11), 2982-2992.

Grahn, S. W., Lowry, A. C., Osborne, M. C., Melton, G. B., Gaertner, W. B., Vogler, S. A., \& Kwaan, M. R. (2019). System-wide improvement for transitions after ileostomy surgery: can intensive monitoring of protocol compliance decrease readmissions? A randomized trial. Diseases of the Colon \& Rectum, 62(3), 363-370.

Hamidi, Y., Moeini, M., \& Yousefi, H. (2018). The effect of an interactive follow-up program on ostomy adjustment of inpatients after their discharge from surgical wards of the hospitals affiliated to Isfahan University of Medical Sciences. International journal of colorectal disease, 33(9), $1295-1297$.

Harris, M. S., Kelly, K., \& Parise, C. (2020). Does preoperative ostomy education decrease anxiety in the new ostomy patient? A quantitative comparison cohort study. Journal of Wound Ostomy \& Continence Nursing, 47(2), 137-139.

Heerschap, C., \& Butt, B. (2021). Algorithmic approaches to ostomy management: An integrative review. Nursing Open, 8(6), $2912-2921$.

Hendren, S., Hammond, K., Glasgow, S. C., Perry, W. B., Buie, W. D., Steele, S. R., \& Rafferty, J. (2015). Clinical practice guidelines for ostomy surgery. Diseases of the Colon \& Rectum, 58(4), 375-387.

Hsu, M. Y., Lin, J. P., Hsu, H. H., Lai, H. L., \& Wu, Y. L. (2020). Preoperative stoma site marking decreases stoma and peristomal complications: a metaanalysis. Journal of Wound Ostomy \& Continence Nursing, 47(3), 249-256.

Ilyas, M. I. M., Haggstrom, D. A., Maggard-Gibbons, M. A., Wendel, C. S., Rawl, S., Schmidt, C. M., \& Krouse, R. S. (2018). Patients with temporary ostomies: veterans administration hospitals multi-institutional retrospective study. Journal of Wound Ostomy \& Continence Nursing, 45(6), 510-515.

Karabulut, H. K., Dinç, L., \& Karadag, A. (2014). Effects of planned group interactions on the social adaptation of individuals with an intestinal stoma: a quantitative study. Journal of clinical nursing, 23(19-20), 2800-2813.

Kerr, N. (2015). Ostomate-for-a-day: A novel pedagogy for teaching ostomy care to baccalaureate nursing students. Journal of Nursing Education, 54(8), 445449. 
Kim, Y. M., Jang, H. J., \& Lee, Y. J. (2021). The effectiveness of preoperative stoma site marking on patient outcomes: A systematic review and metaanalysis. Journal of Advanced Nursing, 77(11): 4332-4346.

Krebsgesellschaft, D., \& Deutsche Krebshilfe, A. W. M. F. (2019). S3-Leitlinie Kolorektales Karzinom, Langversion 1.0, AWMF Registrierungsnummer: 021007OL. Im Internet: http://leitlinienprogramm-onkologie. de/leitlinien, 7.

Krouse, R. S., Grant, M., McCorkle, R., Wendel, C. S., Cobb, M. D., Tallman, N. J., \& Hornbrook, M. C. (2016). A chronic care ostomy self-management program for cancer survivors. Psycho-oncology, 25(5), 574-581.

McGee, M. F. (2016). Stomas. Jama, 315(18), 2032-2032.

Melnyk, B. M., Gallagher-Ford, L., Long, L. E., \& Fineout-Overholt, E. (2014). The establishment of evidence-based practice competencies for practicing registered nurses and advanced practice nurses in real-world clinical settings: Proficiencies to improve healthcare quality, reliability, patient outcomes, and costs. Worldviews on Evidence-Based Nursing, 11(1), 5-15.

Millard, R., Cooper, D., \& Boyle, M. J. (2020). Improving Self-Care Outcomes in Ostomy Patients via Education and Standardized Discharge Criteria. Home healthcare now, 38(1), 16-23.

Moura, L. K. B., Sousa, A. F. L., Nascimento, G. C., \& Queiroz AAFLN, S. D. (2015). Biosafety measures in dental procedures: an integrative review. J Nurs UFPE on line [Internet], 9(10), 1537-44.

Murken, D. R., \& Bleier, J. I. (2019). Ostomy-related complications. Clinics in colon and rectal surgery, 32(03), 176-182.

Nafees, B., Størling, Z. M., Hindsberger, C., \& Lloyd, A. (2018). The ostomy leak impact tool: development and validation of a new patient-reported tool to measure the burden of leakage in ostomy device users. Health and quality of life outcomes, 16(1), 1-12.

Nascimento, M. V. F., da Vera, S. O., Silva, M. C. R., de Morais, F. F., Andrade, E. M. L. R., \& Bastos, S. N. M. A. N. (2018). Perfil sociodemográfico e clínico de pacientes em pós-operatório de confecção de estomas intestinais de eliminação. Cienc. enferm, 24(15), 1-13.

O'Flynn, S. K. (2018). Care of the stoma: complications and treatments. British journal of community nursing, 23(8), 382-387.

Ouzzani, M., Hammady, H., Fedorowicz, Z., \& Elmagarmid, A. (2016). Rayyan—a web and mobile app for systematic reviews. Systematic reviews, 5(1), 1-10.

Page, M. J., McKenzie, J. E., Bossuyt, P. M., Boutron, I., Hoffmann, T. C., Mulrow, C. D., \& Moher, D. (2021). The PRISMA 2020 statement: an updated guideline for reporting systematic reviews. Bmj, 372 .

Pereira, A. P. D. S., Cesarino, C. B., Martins, M. R. I., Pinto, M. H., \& Netinho, J. G. (2012). Associations among socio-demographic and clinical factors and the quality of life of ostomized patients. Revista latino-americana de enfermagem, 20(1), 93-100.

Pouresmail, Z., Nabavi, F. H., Abdollahi, A., Shakeri, M. T., \& Saki, A. (2019). Effect of Using a Simulation Device for Ostomy Self-care Teaching in Iran: A Pilot, Randomized Clinical Trial. Wound management \& prevention, 65(6), 30-39.

Ripollés-Melchor, J., Varela, M. L. D. F., Camargo, S. C., Fernández, P. J., Barrio, Á. C. D., Martínez-Hurtado, E., \& Calvo-Vecino, J. M. (2018). Enhanced recovery after surgery protocol versus conventional perioperative care in colorectal surgery. A single center cohort study. Revista brasileira de anestesiologia, 689(4), 358-368.

Sarabi, N., Navipour, H., \& Mohammadi, E. (2017). Relative tranquility in ostomy patients' social life: A qualitative content analysis. World journal of surgery, 41(8), 2136-2142.

Seo, H. W. (2019). Effects of the frequency of ostomy management reinforcement education on self-care knowledge, self-efficacy, and ability of stoma appliance change among Korean hospitalised ostomates. International wound journal, 16, 21-28.

Silva, K. A. da ., Azevedo, P. F. ., Olimpio, R. de J. J. ., Oliveira, S. T. S. de ., \& Figueiredo, S. N. . (2020). Colostomia: a construção da autonomia para o autocuidado. Research, Society and Development, 9(11), e54391110377.

Sobrado Junior, C. W., Guzela, V. R., Sobrado, L. F., Nahas, S. C., \& Cecconello, I. (2020). Local treatment of colostomy prolapse with the MESH STRIP technique: A novel and highly efficient day hospital technique. Clinics, 75.

Suh, S. R., \& Lee, M. K. (2017, July). Effects of Nurse-Led Telephone-Based Supportive Interventions for Patients With Cancer: A Meta-Analysis. Oncology nursing fórum, 44(4):E168-E184.

Thorpe, G., McArthur, M., \& Richardson, B. (2014). Healthcare experiences of patients following faecal output stoma-forming surgery: A qualitative exploration. International journal of nursing studies, 51(3), 379-389.

Ursi, E. S., \& Galvão, C. M. (2006). Perioperative prevention of skin injury: an integrative literature review. Revista latino-americana de enfermagem, 14(1), $124-131$.

Warrens, M. J. (2021). Kappa coefficients for dichotomous-nominal classifications. Advances in Data Analysis and Classification, 15(1), 193-208.

Wasserman, M. A., \& McGee, M. F. (2017). Preoperative considerations for the ostomate. Clinics in colon and rectal surgery, 30(03), 157-161.

Wen, S. L., Li, J., Wang, A. N., Lv, M. M., Li, H. Y., Lu, Y. F., \& Zhang, J. P. (2019). Effects of transtheoretical model-based intervention on the selfmanagement of patients with an ostomy: A randomised controlled trial. Journal of clinical nursing, 28(9-10), 1936-1951.

Wennström, B., Johansson, A., Kalabic, S., E-son Loft, A. L., Skullman, S., \& Bergh, I. (2020). Patient experience of health and care when undergoing colorectal surgery within the ERAS program. Perioperative Medicine, 9, 1-12. 
Research, Society and Development, v. 11, n. 2, e11211225496, 2022

(CC BY 4.0) | ISSN 2525-3409 | DOI: http://dx.doi.org/10.33448/rsd-v11i2.25496

Windrum, P., García-Goñi, M., \& Coad, H. (2016). The impact of patient-centered versus didactic education programs in chronic patients by severity: the case of type 2 diabetes mellitus. Value in Health, 19(4), 353-362.

Wound, Ostomy and Continence Nurses Society. (2018). WOCN Society Clinical Guideline. Journal of Wound, Ostomy and Continence Nursing, 45(1), 50-58.

Xia, L. (2020). The effects of continuous care model of information-based hospital-family integration on colostomy patients: a randomized controlled trial. Journal of Cancer Education, 35(2), 301-311.

Zelga, P., Kluska, P., Zelga, M., Piasecka-Zelga, J., \& Dziki, A. (2021). Patient-related factors associated with stoma and peristomal complications following fecal ostomy surgery: a scoping review. Journal of Wound, Ostomy and Continence Nursing, 48(5), 415-430.

Zhang, J. E., Wong, F. K. Y., You, L. M., Zheng, M. C., Li, Q., Zhang, B. Y., \& Liu, J. L. (2013). Effects of enterostomal nurse telephone follow-up on postoperative adjustment of discharged colostomy patients. Cancer Nursing, 36(6), 419-428.

Zhang, Y., Xian, H., Yang, Y., Zhang, X., \& Wang, X. (2019). Relationship between psychosocial adaptation and health-related quality of life of patients with stoma: A descriptive, cross-sectional study. Journal of clinical nursing, 28(15-16), 2880-2888. 